\title{
Modelagem Numérica e análise experimental de um protótipo didático de motor Stirling
}

\section{Numerical modeling and experimental analysis of a didactic prototype Stirling engine}

\author{
Laura Vitória Rezende Dias \\ Universidade Federal de Goiás - UFG, Goiânia, GO \\ engenheiralaura@gmail.com \\ Antônio César Baleeiro Alves \\ Universidade Federal de Goiás - UFG, Goiânia, GO \\ abaleeiro@gmail.com
}

\begin{abstract}
Resumo: Este trabalho consiste no desenvolvimento de um protótipo didático de motor Stirling, que foi concebido com o intuito de compreender a máquina principalmente em relação às partes componentes, as dificuldades construtivas e os tipos de parâmetros relevantes para o desempenho. Partindo de dados geométricos e termodinâmicos do protótipo, com aplicação da modelagem de Schmidt, elaborou-se um programa digital que permite calcular grandezas como potência de saída e rendimento. Empregando o protótipo, foram também realizados ensaios em bancada de laboratório. Os resultados obtidos pelos ensaios mostraram que o protótipo alcança velocidade de rotação de até $1.200 \mathrm{rpm}$ a uma temperatura de 159 graus Celsius da fonte térmica externa ao motor, com potência de saída de 100 miliwatt. A comparação entre a simulação computacional e os resultados dos ensaios em bancada demonstrou a influência dos dados construtivos do protótipo sobre a potência de saída e o rendimento do protótipo.
\end{abstract}

Palavras-chave: motor Stirling; modelo isotérmico de Schmidt; energia renovável; motor de combustão externa.

Abstract: This work is the development of a prototype for Stirling engine which was designed with the aim of understanding the machine mainly about the component parts, construction difficulties and type of relevant parameters for its performance. Starting from geometric and thermodynamic data from the prototype, with application of the Schmidt modeling, a digital program was developed to calculate values such as power output and efficiency. The prototype was also used to carryd out bench tests. The experiments have shown that the prototype can reach a rotation speed up to $1.200 \mathrm{rpm}$ at 159 degrees Celsius of external engine heat source, with output power of 100 milliwatt. The comparison between results from simulations and the bench tests demonstrated the great influence of prototype construction data on the output power and the performance of the equipment.

Key words: stirling engine; isothermal model of Schmidt; renewable energy; external combustion engine. 
Revista Ciências Exatas e Naturais, Vol.20, nº.1, Jan/Jun, 2018

\section{Introdução}

Este artigo tem origem na pesquisa e desenvolvimento de um protótipo de um motor Stirling para finalidade, a princípio, didática. A motivação principal da pesquisa está em compreender o funcionamento da máquina, e para isto a metodologia adotada compreende três frentes de ação: explorar os conceitos de Termodinâmica que definem o princípio de funcionamento do dispositivo; detalhar/projetar seus componentes e, por fim, submetê-lo a testes em bancada e por meio de computador. Fontes energéticas que causam impactos ambientais e requerem vultosos investimentos vêm sendo questionadas por diversos segmentos da humanidade, como as formas de aproveitamento energético centralizadas que são muitas vezes de alto custo tanto do ponto de vista econômico quanto ambiental. Em contraposição, destacam-se na atualidade, seja para força motriz ou geração elétrica, formas de aproveitamento distribuídas com utilização de fontes renováveis. Nesse cenário, as explorações de fontes eólicas e solares têm crescido significativamente em todo o mundo. Simultaneamente a esse fenômeno, pesquisas e investimentos em eficiência energética também crescem.

Uma das linhas de pesquisa que integra esses esforços é a redescoberta de máquinas que até recentemente estavam relegadas a um plano secundário e que, hoje, podem ser viabilizadas em aplicações específicas. O motor Stirling, inventado no início do século XIX, vem sendo considerado como alternativa para força motriz e geração de energia elétrica em pequena escala para certas aplicações. Um dos principais atrativos do motor Stirling é sua flexibilidade de operação com multicombustíveis, tais como biomassa, energia solar, resíduos industriais, dentre outros [1].

Coletores solares acoplados ao motor Stirling têm sido aplicados na geração de energia elétrica. O sistema de aproveitamento da energia solar é composto por pratos solares que compõem um sistema de eixos que se movem durante o dia para captação máxima da irradiação solar. Espelhos parabólicos são dispostos de forma que concentram os raios solares incidentes em direção a um ponto focal do espelho, o qual chega a atingir aproximadamente $720^{\circ} \mathrm{C}$. Com um rendimento aproximado de $32 \%$, o calor concentrado é aproveitado para acionar um motor Stirling que é estrategicamente situado no ponto focal do espelho. Em seguida, o motor aciona um alternador que gera uma potência de saída em torno de 31,5 kW [2]. Existem motores Stirling que são acionados pela combustão de biomassa e biogás. A utilização dessas soluções em regiões isoladas, cujo fornecimento de energia pela rede elétrica convencional é limitado, favorece a geração de energia elétrica de forma sustentável pelo aproveitamento de fontes renováveis disponíveis no local [3].

Este artigo possui a seguinte estrutura: na seção II são explanados os Fundamentos da Termodinâmica aplicados às máquinas térmicas; a seção III expõe o conceito da análise isotérmica ideal utilizada na simulação computacional do ciclo Stirling; a seção IV registra o processo de construção do protótipo; a seção V apresenta os resultados da simulação do ciclo e a avaliação experimental dos dados coletados pelo sistema de medição eletrônica; e a última seção trata das conclusões finais a respeito da pesquisa.

\section{Fundamentos da Termodinâmica Aplicados às Máquinas Térmicas}

$\mathrm{Na}$ análise termodinâmica, a energia interna do sistema, $d U$, pode ser definida pela transferência líquida de energia estabelecida na fronteira do sistema na forma de calor, $\delta Q$, 
e/ou trabalho, $\delta W$, conforme (1).

$$
d U=\delta Q-\delta W
$$

sendo que a unidade de energia é o joule: $1 \mathrm{Kg} \cdot \mathrm{m}^{2} / \mathrm{s}^{2}$

A Equação (2), proposta por Clapeyron, representa as relações entre as variáveis de estado de um gás ideal e é denominada Equação de Estados dos Gases Perfeitos [4].

$$
p V=n R T
$$

onde a pressão, $p$, é dada em $P a$, o volume, $V$, em $m^{3}$, a quantidade de matéria, $n$, em mol, a constante universal dos gases, $R$, em $J / k g . K$ e a temperatura, $T$, em $K$. Sendo que P $a$ é o símbolo da unidade pascal $\left(1 P a=1 N / m^{2}\right)$ e $K$, o símbolo da unidade kelvin.

\subsection{Ciclo Stirling}

O ciclo Stirling ideal caracteriza uma máquina térmica de ciclo fechado e regenerativo, ou seja, o gás de trabalho permanece confinado em um espaço hermeticamente fechado (cilindro) e o sistema de regeneração absorve e cede calor ao gás durante o ciclo evitando as demais perdas de energia. No ciclo ocorre absorção e rejeição de calor com o deslocamento do gás pelos permutadores de calor de aquecimento e de arrefecimento que representam, respectivamente, os reservatórios de fontes quente e fria como na máquina térmica de Carnot. Em condições ideais, o ciclo Stirling possui o mesmo rendimento térmico do ciclo de Carnot [3].

O sistema termodinâmico no motor Stirling é o gás que fica confinado na câmara de expansão. Pela fronteira entre a câmara onde fica armazenado o gás e a vizinhança ocorre o fluxo de energia térmica e a realização do trabalho. O gás de trabalho passa por processos cíclicos de expansão, resfriamento, compressão e aquecimento a partir da energia calorífica proveniente da fonte de calor externa. A Figura 1 apresenta o diagrama de pressão versus volume, $p \times V$, cuja área representa o trabalho líquido do ciclo $\left(W_{l i q}\right)$.

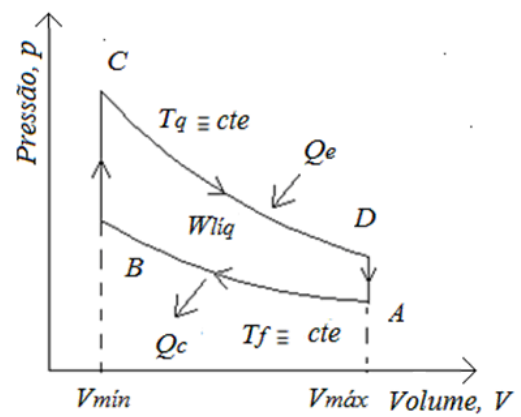

Figura 1. Diagramas $p \times V$ do ciclo Stirling

De acordo com a primeira lei da Termodinâmica, a quantidade de calor transferido pela fonte de calor ao gás durante o processo de expansão, $Q e$, é igual ao trabalho, $W e$, realizado sobre o pistão, e a quantidade de calor rejeitado pelo gás para a fonte fria, Qc, é igual ao trabalho realizado pelo pistão sobre o gás, Wc, durante o processo de compressão [5]. 
Revista Ciências Exatas e Naturais, Vol.20 , nº.1, Jan/Jun, 2018

\section{Motor Stirling}

O motor Stirling é constituído basicamente por duas câmaras e dois pistões, além de sistemas mecânicos para transmissão de movimento e conjugado. Dentro das câmaras está confinado o gás de trabalho que passa por processos termodinâmicos a partir da energia calorífica proveniente de uma fonte de calor externa. O deslocamento do gás confere movimento alternado entre os pistões que transmitem um momento de força para o virabrequim. Dessa forma, a energia térmica é convertida em energia mecânica.

Métodos de análise termodinâmica do ciclo Stirling têm sido desenvolvidos para modelagem do funcionamento do motor. Esta seção apresenta uma descrição da modelagem de Schmidt que foi escolhida por sua simplicidade de aplicação.

\subsection{Modelagem e Análise de Motor Stirling}

Schmidt desenvolveu uma análise do ciclo termodinâmico do motor Stirling baseada em soluções analíticas e condições ideais. As equações de Schmidt permitem estimar grandezas como o trabalho líquido do ciclo e a potência do motor.

A Figura 2 apresenta um esquema do motor Stirling tipo gama mostrando as principais variáveis da modelagem de Schmidt, onde $V c, V f, V r, V q$ e $V e$ são os volumes dos espaços de compressão, do permutador arrefecedor, do regenerador, do permutador aquecedor e do espaço de expansão, respectivamente e, $T f, T q, T e$ e $T c$ são as temperaturas nos espaços do permutador arrefecedor, do permutador aquecedor, do espaço de expansão e do espaço de compressão, respectivamente. Sendo que os volumes são dados em $m^{3}$ e as temperaturas, em $K$.

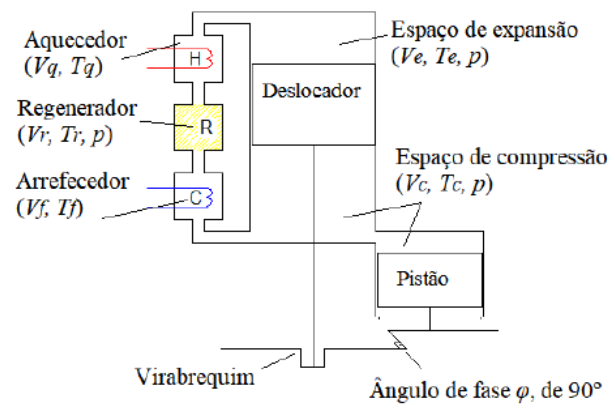

Figura 2. Esquema de um motor Stirling tipo Gama

A análise isotérmica de Schmidt pressupõe que as temperaturas do espaço de expansão, $T e$, e do espaço do permutador aquecedor, $T q$, encontram-se sob a mesma temperatura, bem como as temperaturas do espaço de compressão, $T c$, e do espaço do permutador arrefecedor, Tf $[6]$.

A Equação (3) permite obter o volume do espaço de expansão, $V_{e}$, em função do volume deslocado pelo êmbolo deslocador, $V_{d e}$, o volume morto do espaço de expansão, $V_{m e}$, e o ângulo de rotação, $\theta$, do virabrequim.

$$
V_{e}=0,5 V_{d e}(1-\cos \theta)+V_{m e}
$$

O volume do espaço de compressão é calculado em função do volume deslocado pelo pistão de trabalho, $V_{d c}$, o volume morto do espaço de compressão, $V_{m c}$, o ângulo de giro do vira- 
brequim, $\theta$, e ângulo de fase, $\varphi$, medido entre o deslocador e o pistão de trabalho, onde $\phi$ equivale a $90^{\circ}$, conforme [4].

$$
V_{c}=0,5 V_{d e}(1-\cos \theta)+0,5 V_{d c}[1-\cos (\theta-\varphi)]+V_{m c}
$$

O volume total é dado por (5), sendo que $V_{r}$ é o volume do regenerador.

$$
V=V_{e}+V_{r}+V_{c}
$$

A Equação (6) apresenta a pressão do gás no ciclo, $p$, que é expressa em função da pressão média do gás, $p_{\text {media }}$. Sendo que a pressão média é definida pelas pressões máxima e mínima do gás nos processos de expansão e compressão, respectivamente.

$$
p=\frac{p_{\text {media }} \sqrt{\left(1-c^{2}\right.}}{1-c \cos \theta-a},
$$

sendo:

$$
\begin{array}{r}
\frac{p_{\text {min }}}{p_{\text {media }}}=\sqrt{\frac{1-c}{1+c}}, \\
\frac{p_{\text {max }}}{p_{\text {media }}}=\sqrt{\frac{1+c}{1-c},} \\
c=\frac{B}{S} \\
a=\arctan \frac{v \sin \varphi}{t+\cos \varphi+1} \\
B=\sqrt{t^{2}+2(t-1) v \cos \varphi+v^{2}-2 t+1} \\
S=t+2 t+X_{d e}+\frac{4 t X_{r}}{1+t}+v+2 X_{d c}+1
\end{array}
$$

Os parâmetros $c, a, B$ e $S$ dependem diretamente das relações de temperaturas das fontes quente e fria, $t$, dos volumes deslocados pelo pistão e pelo deslocador, $v$, e dos volumes mortos presentes no motor, $X_{d e}, X_{d c}$, e $X_{r}$. Os volumes mortos presentes no motor são definidos como os espaços ocupados pelo gás que não passam por expansão e compressão como os volumes dos permutadores de calor, regenerador e dos condutos que interligam os cilindros [7].

O trabalho líquido do sistema durante o ciclo é resultante da soma algébrica do trabalho realizado nos espaços de compressão e de expansão, e é expresso por (13).

$$
W_{\text {liq }}=\frac{p_{\text {media }} V_{\text {de }} \pi c \sin a}{1+\sqrt{1-c^{2}}}-\frac{p_{\text {media }} V_{d e} \pi c t \sin a}{1+\sqrt{1-c^{2}}}
$$

As Equações (14) e (15) são utilizadas para calcular a potência e o rendimento do motor.

$$
\begin{gathered}
P=n W_{l i q} \\
\eta=\frac{W_{l i q}}{W_{e}}
\end{gathered}
$$

sendo que a potência, $P$, é calculada em função do trabalho líquido do ciclo, $W_{l i q}$, e da velocidade angular de rotação do volante, $n$, e o rendimento, $\eta$, expressa o quanto da energia fornecida ao sistema foi convertida em trabalho. O volante é um componente mecânico que armazena energia cinética de rotação e amortece os impulsos provocados pelos pistões sobre o virabrequim. 
Revista Ciências Exatas e Naturais, Vol.20 , nº.1, Jan/Jun, 2018

\section{Desenvolvimento de um protótipo e sistema eletrônico de aquisição de dados}

Ao longo do desenvolvimento da pesquisa construiu-se o protótipo utilizando técnicas, processos de fabricação e materiais adequados para usinagem do cabeçote e o bloco do motor. A Figura 3 mostra uma visão em perspectiva dos principais componentes do protótipo.

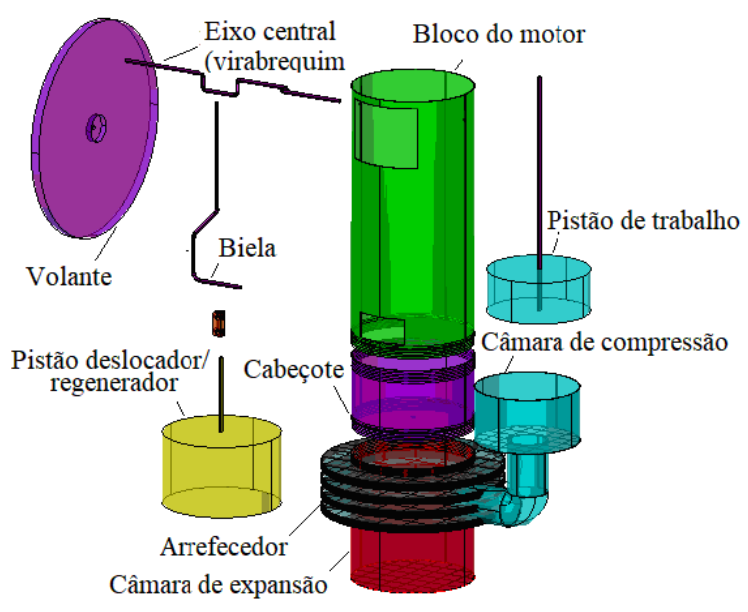

Figura 3. Visão em perspectiva do protótipo.

Visando proporcionar um maior gradiente de temperatura entre a zona quente e a zona fria do cilindro de expansão optou-se por fazê-lo com um permutador de arrefecimento constituído de um reservatório para água fria. Além disso, o cilindro de expansão foi construído utilizando uma lata de aço laminado cujas paredes apresentam menor espessura (conforme a Figura 4).

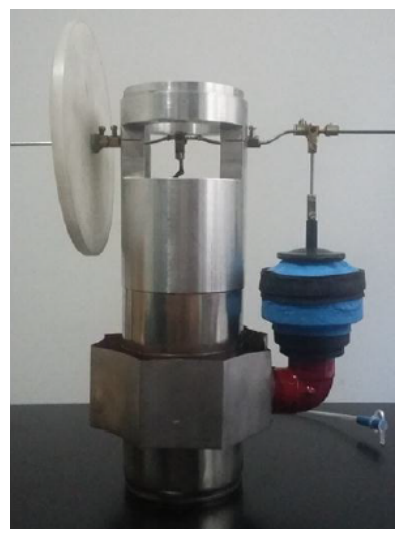

Figura 4. Fotografia do protótipo finalizado

A base inferior do cabeçote encaixa-se na parte superior da câmara de aquecimento como se fosse uma tampa. No entanto, a haste do êmbolo deslocador passa pelo cabeçote para ser 
interligado a biela. Este é um ponto delicado do motor, uma vez que pode ocorrer fugas do fluido de trabalho.

O deslocador (êmbolo) é confeccionado com lã de aço. Dessa forma, ele contribui com uma ação regenerativa para armazenamento do calor. O pistão de trabalho é interligado a um dispositivo tipo diafragma (compressor de membrana de borracha).

A Figura 5 mostra detalhes do mecanismo de abertura presente na parte superior do bloco para passagem do eixo de manivelas. Esse mecanismo possibilitou a redução da vibração demasiada que ocorria com o eixo de manivelas.
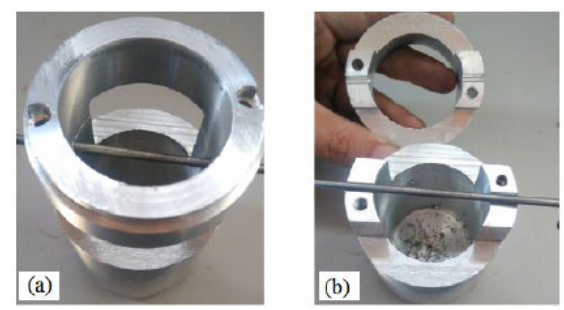

Figura 5. a) Bloco e b) Mecanismo de abertura superior do bloco do motor para encaixe do virabrequim

Utilizou-se o combustível querosene como fonte de calor para aquecer a superfície do permutador de aquecimento por meio de uma lamparina. Usando um tacômetro digital verificou-se que este protótipo alcançou uma velocidade angular de $1.140 \mathrm{rpm}$.

\subsection{Instrumentação do motor}

A estratégia utilizada para instrumentação do motor consiste na utilização de sensores para coleta de dados, como a velocidade angular de rotação do volante. Proposto por Conner, o método fundamenta-se na dinâmica do movimento de rotação e visa determinar a potência de atrito dos componentes móveis do motor por meio da desaceleração do volante [8].

Para medição das grandezas angulares de rotação do volante do protótipo, bem como as temperaturas médias das zonas quente e fria do espaço de trabalho, foram utilizados sensores e uma placa eletrônica com um microcontralador ATMega328 da ATMEL, o Arduino Uno $[9]$.

Sensores de temperatura LM35 foram afixados nas superfícies dos permutadores de calor de aquecimento e de arrefecimento para coleta das temperaturas $T q$ e $T f$ [10].

O método de Conner não faz medições da temperatura dentro dos espaços de expansão e de compressão, mas somente da temperatura nas superfícies dos permutadores de calor. Sendo assim, utilizou-se o sensor termopar tipo $K$, para medição da temperatura dentro da câmara de expansão. Para medição do período entre as revoluções do volante utilizou-se o sensor de efeito Hall US1881.

\subsubsection{Dinâmica do movimento de rotação aplicado ao volante}

O movimento rotacional do volante ocorre em virtude do momento da força (torque) aplicada pelo pistão de trabalho ao eixo de manivelas. Esse torque promove uma aceleração angular ao volante [11]. Por meio do sistema eletrônico de aquisição de dados, os períodos entre as revoluções do volante foram coletados e utilizados na Equação (16) para determinação 
Revista Ciências Exatas e Naturais, Vol.20 , nº.1, Jan/Jun, 2018

da velocidade angular do volante em rotações por segundo.

$$
n=\frac{1}{T}
$$

onde $n$ é a velocidade angular em rps e $T$, o período de revolução em $s$.

A energia cinética provém do movimento de rotação do volante que possui uma inércia rotacional. Essa inércia depende da distribuição de massa com relação ao eixo de rotação. Assim, calcula-se a energia cinética de rotação pela Equação (17).

$$
E_{c}=\frac{I \omega^{2}}{2}
$$

onde $E_{c}$ é a energia cinética em joule $(J), I$, o momento de inércia em $\mathrm{kg} \cdot \mathrm{m}^{2}$ e $\omega$, a velocidade angular em $\mathrm{rad} / \mathrm{s}$.

O cálculo do momento de inércia para um disco cilíndrico maciço é calculado pela Equação (18).

$$
I=\frac{M R^{2}}{2}
$$

onde $M$ é a massa do disco em $\mathrm{kg}$ e $R$, o raio do disco em $m$.

Pelo Teorema da Energia Cinética, quando o torque realiza trabalho em um corpo, a energia cinética de rotação do corpo muda de forma proporcional ao trabalho feito. Assim, o trabalho pode ser definido como a variação de energia cinética de rotação do corpo, conforme (19).

$$
W=\frac{I \omega_{f}^{2}}{2}-\frac{I \omega_{i}^{2}}{2}
$$

onde $W$ é o trabalho em $J, \omega_{i}$, a velocidade angular inicial e $\omega_{f}$, a velocidade angular final, em $\mathrm{rad} / \mathrm{s}$.

A magnitude da potência transmitida pelo eixo do motor é caracterizada pela relação entre o trabalho e o tempo, conforme mostra a Equação (20).

$$
P=\frac{d W}{d t}
$$

onde $P$ é a potência instantânea dada em $W$. Sendo que $W$ é o símbolo para watt( $1 W=$ $1 \mathrm{~J} / \mathrm{s})$.

\section{Resultados e Discussões}

Uma simulação computacional das equações de Schmidt aplicadas ao protótipo foi realizada e utilizada como referência para a análise das informações coletadas pelo sistema de aquisição de dados durante os testes de operação realizados com o protótipo.

\subsection{Simulação Computacional do Ciclo Stirling do Protótipo}

O método isotérmico de Schmidt possibilita a simulação do ciclo Stirling ideal e serve como ponto de partida para investigação do comportamento do motor com relação às variáveis que são utilizadas para descrever sua operação. Assim, realizou-se uma simulação das equações de Schmidt em ambiente de programação MATLAB, tendo como dados de 
Tabela 1. Dados de Entrada da Simulação de Schmidt

\begin{tabular}{ccc}
\hline Volumes & Símbolos & Valores $\left(\mathrm{cm}^{3}\right)$ \\
\hline Volume deslocado do espaço de expansão & $V_{d e}$ & 62,35 \\
Volume deslocado do espaço de compressão & $V_{d c}$ & 17,59 \\
Volume morto do espaço de expansão & $V_{m e}$ & 33,26 \\
Volume morto do espaço de compressão & $V_{m c}$ & 88,24 \\
Volume do regenerador & $V_{r}$ & 93,52 \\
\hline
\end{tabular}

entrada as dimensões e as condições de operação do protótipo. A Tabela 1 apresenta os valores dos dados de entrada da simulação. Os valores volumétricos apresentados na tabela foram obtidos conforme as dimensões do protótipo.

As condições de operação do protótipo também são declaradas como dados de entrada na simulação. A Tabela 2 apresenta os valores das temperaturas médias dos espaços de expansão e de compressão e a velocidade angular de rotação do volante. Esses valores foram obtidos por meio do sistema eletrônico de aquisição de dados.

Tabela 2. Variáveis Referentes as Condições de Operação

\begin{tabular}{ccc}
\hline Variáveis & Símbolos & Valores \\
Temperatura do espaço de expansão & $T_{e}$ & $417,78 \mathrm{~K}$ \\
Temperatura do espaço de compressão & $T_{c}$ & $340,98 \mathrm{~K}$ \\
Velocidade angular do volante & $n$ & $1.140 \mathrm{rpm}$ \\
\hline
\end{tabular}

A Tabela 3 mostra os resultados das variáveis de saída obtidos pela simulação: o trabalho líquido, $W_{\text {liq }}$, a potência, $P$, e o rendimento, $\eta$, de acordo com o modelo proposto por Schmidt.

Tabela 3. Resultados da Simulação para o Protótipo

\begin{tabular}{ccc}
\hline Variáveis & Símbolos & Valores \\
Trabalho líquido & $W_{l i q}$ & $11,10 \mathrm{~mJ}$ \\
Potência & $P$ & $211,00 \mathrm{~mW}$ \\
Rendimento & $\eta$ & $18,38 \%$ \\
\hline
\end{tabular}

A partir da simulação é possível constatar o quanto as condições de operação influenciam o rendimento e a potência do motor, assim como as características geométricas dos cilindros e dos pistões. Deste modo, as variáveis volumétricas como os volumes dos permutadores podem ser reajustadas de modo a reduzir os volumes mortos, bem como a quantidade de calor transferida para o gás pode ser incrementada a partir da otimização dos permutadores de calor.

\subsection{Resultados da Bancada de Ensaios}

A Figura 6 mostra o aparato experimental com os sensores de temperatura LM35 e o sensor de efeito Hall. Os sensores LM35 foram utilizados para medição das temperaturas nas superfícies dos permutadores de calor. 
Revista Ciências Exatas e Naturais, Vol.20, nº.1, Jan/Jun, 2018

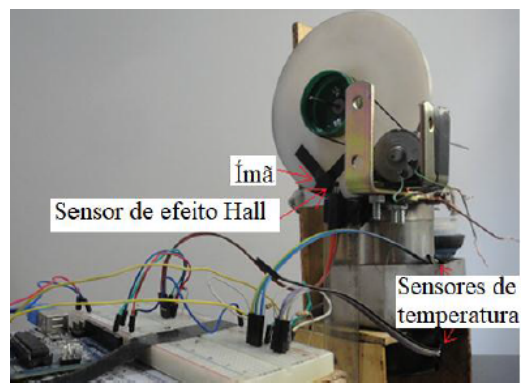

Figura 6. Aparato experimental para medições

A curva da Figura 7 apresenta a evolução da temperatura na superfície do permutador aquecedor, $T q$. Sendo que a temperatura é dada em graus Celsius e o tempo em segundos.

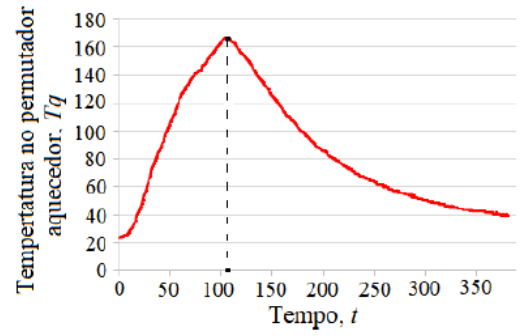

Figura 7. Temperatura na superfície do permutador de aquecimento

Conforme mostra a curva da Figura 7, entre os instantes de $0 s$ e $105 s$ a temperatura se elevou até $167^{\circ} \mathrm{C}$, e depois dos $105 \mathrm{~s}$, houve uma queda devido à retirada da fonte de calor. É interessante destacar que o calor liberado pela queima do querosene não é totalmente transferido para o permutador, pois uma parte desse calor é perdida para o ambiente na região próxima a chama e na própria superfície do permutador.

A curva da Figura 8 apresenta a evolução da temperatura na superfície do permutador de arrefecimento, Tf. O gráfico mostra que a temperatura no permutador atingiu aproximadamente $32^{\circ} \mathrm{C}$.

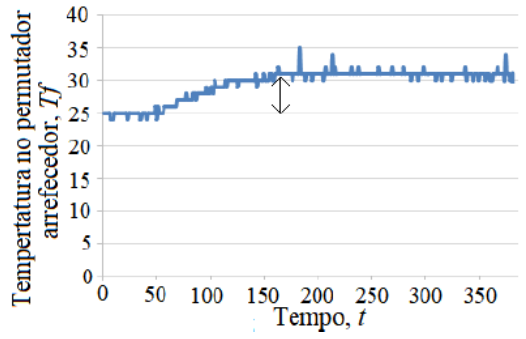

Figura 8. Temperatura na superfície do permutador de arrefecimento

Pelo gráfico da Figura 8 verifica-se que houve um aumento de $6^{\circ} \mathrm{C}$ na parede do permutador de arrefecimento. Como os permutadores de aquecimento e de arrefecimento foram 
instalados no mesmo cilindro, ocorre propagação de calor pela superfície do cilindro.

O gráfico da Figura 9 mostra a temperatura do gás no espaço de expansão, Te, medida pelo termopar. A curva do gráfico demonstra que, entre os instantes de $0 s$ e $105 s$, houve uma elevação da temperatura do gás conforme o aumento da temperatura no permutador de aquecimento (Figura 7). Depois de $105 \mathrm{~s}$, mesmo com a retirada da fonte de calor, a temperatura do gás chegou a $159^{\circ} \mathrm{C}$ e caiu de forma lenta com o tempo.

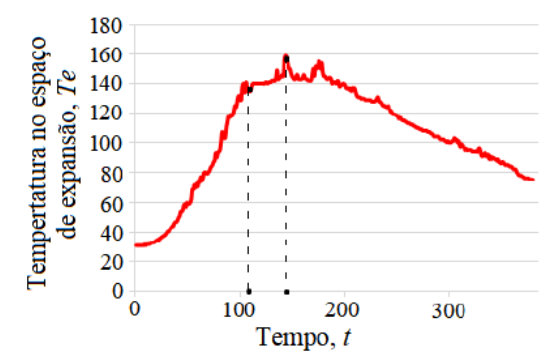

Figura 9. Evolução da temperatura do gás no espaço de expansão

A curva ilustrada na Figura 10 mostra a evolução da temperatura do gás na zona fria da câmara de compressão, $T c$.

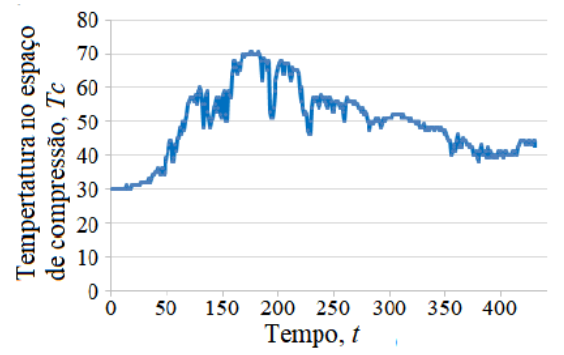

Figura 10. Evolução da temperatura do gás no espaço de compressão

A partir do gráfico da Figura 10 observa-se que a temperatura do gás atinge cerca de $71^{\circ} \mathrm{C}$. Desta forma, observa-se que, $T e<T q$ e $T c>T f$. O que demonstra que o projeto de permutadores de calor é crucial para a transferência eficiente de calor, pois se a temperatura do gás na zona fria fosse mais próxima da temperatura da superfície do permutador arrefecedor, $32^{\circ} \mathrm{C}$ e, a temperatura do gás na zona quente fosse mais próxima da temperatura do permutador aquecedor, $167^{\circ} \mathrm{C}$, o rendimento teórico, pela expressão da eficiência térmica do ciclo de Carnot, seria aproximadamente igual a $30 \%$.

A Figura 11 mostra graficamente a evolução da velocidade angular do volante durante o tempo de operação do motor. Sendo que a velocidade é dada em rotações por minuto.

A partida do motor ocorre aproximadamente $30 s$ depois de igada a fonte de calor. A velocidade angular cresce atingindo $1.200 \mathrm{rpm}$ devido à aceleração angular do volante. Em seguida, entre o tempo aproximado de $53 \mathrm{~s}$ a $105 \mathrm{~s}$, o volante entra em regime estacionário e passa a girar com velocidade aproximadamente constante de $1.140 \mathrm{rpm}$. Após esse tempo, a fonte de calor é retirada e o volante continua a girar com o calor residual. Devido a fontes de friç̧ão o volante desacelera até cessar seu movimento. 
Revista Ciências Exatas e Naturais, Vol.20 , nº.1, Jan/Jun, 2018

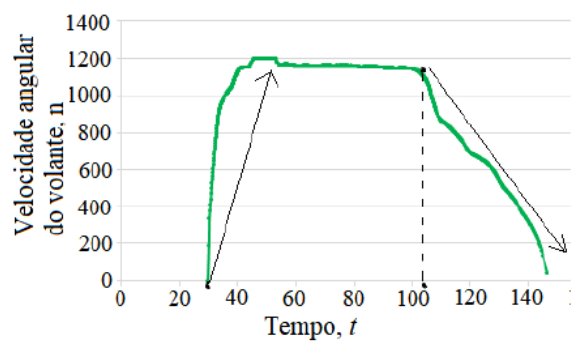

Figura 11. Comportamento da velocidade angular com o tempo

A Figura 12 mostra a curva da energia cinética de rotação do volante durante o tempo em que motor opera. Sendo que a energia cinética foi calculada utilizando-se a Equação (17).

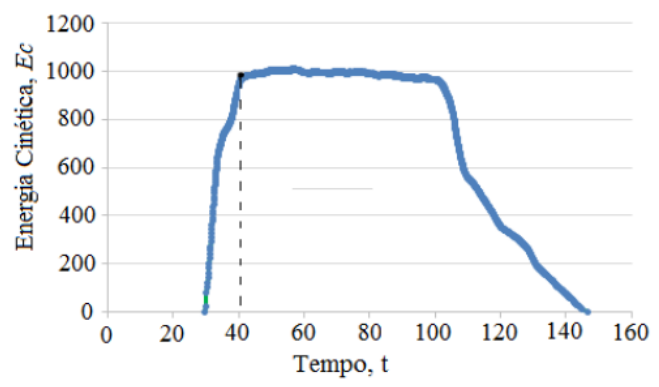

Figura 12. Gráfico da energia cinética com o tempo

No tempo aproximado de $40 \mathrm{~s}$, a energia cinética é cerca de $1000 \mathrm{~mJ}$. Como a velocidade angular inicial do volante é zero, a potência média de saída é em torno de $100 \mathrm{~mW}$.

Dentre os desafios de realizar a análise do ciclo termodinâmico Stirling está a dificuldade de quantificar as perdas que ocorrem durante a operação do motor. As perdas ocorrem devido a fugas de fluido, queda de pressão, perdas de calor e perdas mecânicas por atrito. De modo que, a dificuldade de mensurar essas perdas torna o resultado do rendimento do motor impreciso.

\section{Conclusões}

O processo de construção do protótipo propiciou o enfrentamento e a busca de soluções das dificuldades técnicas construtivas do motor como a necessidade de: garantia de vedação das câmaras, redução de atrito mecânico pelo alinhamento do eixo de manivelas com as hastes do pistão de trabalho e do deslocador, construção de permutador arrefecedor com água para melhorar a dissipação de calor e construção de mancais com baixo atrito para o deslizamento livre do virabrequim.

O desempenho do motor Stirling está relacionado de forma direta com a eficiência dos permutadores de calor. Pela expressão do rendimento de Carnot, obteve-se um rendimento em torno de 18\%, considerando a temperatura do gás nos espaços de compressão e ex- 
pansão (Tabela 3). Se o gás tivesse a mesma temperatura dos permutadores de calor, esse rendimento seria de $30 \%$.

Pela simulação do ciclo Stirling do protótipo estimou-se uma potência de $211 \mathrm{~mW}$. Pela análise da dinâmica rotacional do volante (por meio do sistema de medição) obteve-se uma potência média de saída de aproximadamente $100 \mathrm{~mW}$. A potência estimada pela simulação é duas vezes maior que a potência obtida pelo sistema de medição eletrônica devido as considerações ideais assumidas por Schmidt. O protótipo resultante desta pesquisa, apesar de possuir pequeno porte, permitiu a obtenção de informações importantes que podem abrir caminhos para a construção de motores Stirling de maiores dimensões e consequentemente com valores de potências de saída para utilização prática.

Dentre as sugestões de temas para aprofundamento investigativo do motor Stirling, podese citar:

1. Estudo e modelagem de permutadores de calor de aquecimento e arrefecimento;

2. Análise de perdas de energia térmica e mecânica;

3. Análise de rendimento do protótipo com diferentes combustíveis e

4. Otimização dos volumes mortos do motor para melhorar o desempenho do motor.

\section{Referências}

[1] CERVANTES, J. V. Metodologia para el rediseño de motores de ciclo Stirling. 93 p. Dissertação (Mestrado). Curso de Ingenieria Mecânica, Sección de Estudios de Posgrado e Investigación, Instituto Politécnico Nacional, México, 2006.

[2] HILLIG, T. A Hybrid Solution with Concentrated Solar Power (CSP) and Fuel for Baseload Mining Operations. [S.l.], 2016. 13 p.

[3] COSTA, J. M. S. Metodologia Simplificada para a Análise Termoeconomica de Sistemas de Cogeração como Motor Stirling. 149 p. Dissertação (Mestrado). Universidade do Minho, Escola de Engenharia, 2013.

[4] SOnNTAG, R. E.; BORGNAKKE, C.; WYlen, G. J. V. Fundamentos da Termodinâmica. 6. ed. [S.l.]: Editora Edgard Blucher Ltda, 2003. 584 p.

[5] LLOYD, C. A. A Low Temperature Differential Stirling Engine for Power Generation. 132 p. Dissertação (Mestrado). University of Canterbury, Curso de Engenharia, 2009.

[6] URIELI, I. A Computer Simulation of Stirling Cycle Machines. 313 p. Tese (Doutorado). University of the Witwatersrand, Faculty of Engineering, Curso de Mechanical Engineering, Johannesburg, 1997.

[7] PINILla, A. V. Diseño de un prototipo bio-Stirling para abastecer la demanda energética de una vivienda rural. Valparaiso: [s.n.], 2012.

[8] CONNER, D. How to measure engine friction using flywheel desaceleration?, 2016. Disponível em: http://www.solarheatengines.com/2013/02/17/how-to measureenginefriction-using- flywheel-deceleration/, 2016.

[9] Arduino, 2017. Disponível em: ¡ https://store.arduino.cc/usa/arduino-uno-rev3¿.

[10] LM35 Datasheet, 2017. Disponível em:i http://www.ti.com/lit/ds/symlink/lm35.pdf_. 
Revista Ciências Exatas e Naturais, Vol.20, nº.1, Jan/Jun, 2018

[11] CORRADI, W.; TARSIA, R. D. Fundamentos de Física 1. [S.l.]: Editora UFMG, 2010. 514 p. 